\title{
A pipeline for rapidly generating genetically engineered mouse models of pancreatic cancer using in vivo CRISPR-Cas9-mediated somatic recombination
}

\author{
Noboru Ideno ${ }^{1}$ Hiroshi Yamaguchi ${ }^{1} \cdot$ Takashi Okumura $^{1} \cdot$ Jonathon Huang ${ }^{1} \cdot$ Mitchell J. Brun $^{2} \cdot$ Michelle L. Ho $^{3}$. \\ Junghae Suh $^{3} \cdot$ Sonal Gupta ${ }^{1} \cdot$ Anirban Maitra ${ }^{1} \cdot$ Bidyut Ghosh $^{1}$
}

Received: 27 August 2018 / Revised: 13 November 2018 / Accepted: 2 December 2018 / Published online: 6 February 2019

(c) United States \& Canadian Academy of Pathology 2019

\begin{abstract}
Genetically engineered mouse models (GEMMs) that recapitulate the major genetic drivers in pancreatic ductal adenocarcinoma (PDAC) have provided unprecedented insights into the pathogenesis of this lethal neoplasm. Nonetheless, generating an autochthonous model is an expensive, time consuming and labor intensive process, particularly when tissue specific expression or deletion of compound alleles are involved. In addition, many of the current PDAC GEMMs cause embryonic, pancreas-wide activation or loss of driver alleles, neither of which reflects the cognate human disease scenario. The advent of CRISPR/Cas9 based gene editing can potentially circumvent many of the aforementioned shortcomings of conventional breeding schema, but ensuring the efficiency of gene editing in vivo remains a challenge. Here we have developed a pipeline for generating PDAC GEMMs of complex genotypes with high efficiency using a single "workhorse" mouse strain expressing Cas9 in the adult pancreas under a $p 48$ promoter. Using adeno-associated virus (AAV) mediated delivery of multiplexed guide RNAs (sgRNAs) to the adult murine pancreas of $p 48$-Cre; $L S L$-Cas 9 mice, we confirm our ability to express an oncogenic Kras ${ }^{G 12 D}$ allele through homology-directed repair (HDR), in conjunction with CRISPRinduced disruption of cooperating alleles (Trp53, Lkbl and AridlA). The resulting GEMMs demonstrate a spectrum of precursor lesions (pancreatic intraepithelial neoplasia [PanIN] or Intraductal papillary mucinous neoplasm [IPMN] with eventual progression to PDAC. Next generation sequencing of the resulting murine PDAC confirms HDR of oncogenic $\operatorname{Kras}^{G 12 D}$ allele at the endogenous locus, and insertion deletion ("indel") and frameshift mutations of targeted tumor suppressor alleles. By using a single "workhorse" mouse strain and optimal AAV serotype for in vivo gene editing with combination of driver alleles, we present a facile autochthonous platform for interrogation of the PDAC genome.
\end{abstract}

Supplementary information The online version of this article (https:// doi.org/10.1038/s41374-018-0171-z) contains supplementary material, which is available to authorized users.

Anirban Maitra

amaitra@mdanderson.org

$\triangle$ Bidyut Ghosh

bghosh@mdanderson.org

1 Department of Translational Molecular Pathology and Sheikh Ahmed Pancreatic Cancer Research Center, UT MD Anderson Cancer Center, Houston, TX, USA

2 Department of Chemical and Biomolecular Engineering, Rice University, Houston, TX, USA

3 Department of Bioengineering, Rice University, Houston, TX, USA

\section{Introduction}

Pancreatic ductal adenocarcinoma (PDAC) is one of the most lethal cancers known, due to the absence of reliable early diagnosis and the absence of effective therapeutic regimens [1]. It is currently the third leading cause of cancer worldwide and is projected to become second leading cause of cancer in the United States by 2030. The median 5-year survival rate of PDAC is only 9\% [2], and underscores the need for developing new approaches in understanding the molecular mechanisms underlying the disease.

The advent of next generation sequencing (NGS) and its application as part of large publicly funded efforts such as The Cancer Genome Atlas (TCGA) and International Cancer Genome Consortium (ICGC) has identified a huge catalogue of genes that are altered in PDAC [3, 4], although in many instances, particularly with low frequency 
mutations, the functional consequences of the alterations remain unknown. The definitive evidence for gene perturbation in carcinogenesis is typically obtained via autochthonous models, which in the context of PDAC, is exemplified by expression of a mutant Kras allele in the pancreas alongside one or more cooperating mutations [5]. First developed in 2003, PDAC GEMMs have dramatically accelerated our understanding of the molecular pathogenesis of this neoplasm, including how the genetic background of the cancer impacts the tumor microenvironment (TME) and resulting immune response [6-8]. In contrast to cell line or patient-derived xenograft (PDX) models, autochthonous models harbor the precursor lesions observed in the multistep progression of PDAC, as well as the stromadense TME observed in the corresponding human disease [9-11].

Although autochthonous models have been instrumental in our understanding the molecular underpinnings of PDAC, the conventional $\mathrm{Cre}$-based recombinatorial models do not faithfully mimic adult onset human PDAC due to their inherent limitation of inducing Ras activation and cooperating gene alteration(s) in every epithelial cell of the pancreas during development. In some respects, the conventional models are more analogous to "familial" PDAC where every cell in the pancreas is predisposed to neoplasia due to a germline anomaly (for example, CDKN2A/p16), and cancers arise due to stochastic bi-allelic gene inactivation $[12,13]$. In addition to this conceptual limitation, conventional $\mathrm{Cre}$-mediated recombinatorial models are also hindered by time, labor and expenses required for generating appropriate numbers of colonies, especially with complex genotypes where the "pups" of interest might only be a minor fraction of the litter. A timeframe of 12-18 months for completing the breeding of complex mouse genotypes is not unusual in the field. Novel platforms that bypass the pancreas-wide embryonic expression of mutant Kras and loss of cooperating alleles, while also greatly improving the efficiency of model generation would be a significant advancement in the preclinical arena.

Recently, the RNA-guided endonuclease Cas 9 from microbial type II CRISPR (clustered regularly interspaced short palindromic repeat) system has emerged as a powerful tool for genome engineering in mammalian cells [14-16], including somatic cell editing in a variety of mouse organs that includes brain, lung, liver and pancreas [17-21]. CRISPR/Cas9 can be targeted to the desired genomic loci by using programmable 20-bp single guide RNAs (sgRNAs) to generate DNA double stranded breaks which induce genome editing via one of the two DNA damage repair pathways: non-homologous end-joining (NHEJ) resulting in insertion-deletion (INDELs), or homologydirected repair (HDR) resulting in precise sequence substitution in the presence of a repair template [22-25]. The multiplexing abilities of gene editing by combining multiple sgRNAs to the Cas9 provides this programmable nuclease system with a unique advantage for conducting in vivo combinatorial gene editing, especially in the context of developing autochthonous cancer models [21]. There have been several approaches used for delivering CRISPR/Cas 9 in vivo in murine pancreatic cells, including lentivirus and plasmid based transfection [26, 27], although many of these vectors are hindered by low efficiency of multiplexed gene editing and risk of on-target recombination in unintended cell types or tissues [28]. In recent years, adeno-associated virus (AAV) vectors, in particular, have been increasingly utilized for in vivo delivery approaches of sgRNAs due to their efficiency, lack of inherent pathogenicity and strong safety profile [29-32].

In order to generate a "workhorse" platform using a genetically engineered mice that develop PDAC with a variety of complex genotypes, we have developed a simple injection method by employing AAV-based delivery of sgRNAs cargo to the pancreas of adult mice, where Cas 9 activity is restricted to the p48-expressing acinar compartment [33]. Our model overcomes several challenges of the current non inducible Cre-based models, including the introduction of mutant alleles in a subset of adult pancreatic epithelial cells (rather than pancreas-wide embryonic expression), and the use of a single "workhorse" mouse genotype ( $p 48$-Cre; LSL-Cas9) from which cancers harboring multiple complex genotypes can be readily generated through somatic gene editing, thus greatly increasing efficiency. Importantly, in contrast to some of the recently described CRISPR/Cas9-mediated PDAC models that require an oncogenic $\mathrm{Kras}^{\mathrm{G} 12 \mathrm{D}}$ allele in the pancreas on which cooperating mutations are then introduced by CRISPR/Cas9 [26, 27], our method dispenses with the need for a constitutive $\operatorname{Kras}^{\mathrm{G} 12 \mathrm{D}}$ allele, by including a mutant $\operatorname{Kras}^{\mathrm{G} 12 \mathrm{D}}$ template in the delivery cargo that recombines with, and replaces, the endogenous wild type allele through HDR.

\section{Materials and methods}

\section{Generation of pancreas specific Cre-dependent Cas9 mice}

Conditional Cas9 mice (B6J.129 (B6N)-Gt (ROSA) 26 Sor $\left.^{\text {tml } 1(C A G \text {-cas } 9 *-E G F P) F e z h} / \mathrm{J}\right)$ was obtained from the Jackson Laboratory (Stock \# 026175) and was crossed with pancreas specific $p 48$-Cre mice in order to generate experimental p48-Cre; LSL-Cas 9 mice. The following primers were used to genotype Cas9 (Cas9_Common-S 5'-AAGGGAGCTGCAGTGGAGTA-3'; Cas9_Wild-AS 5'-CCGAAAATCTGTGGGAAGTC-3'; Cas9_Mut-AS 
5'-CGGGCCATTTACCGTAAGTTAT-3') and p48Cre (ptfla_Fw 5'-AACCAGGCCCAGAAGGTTAT-3'; ptf1a_Rv 5'-TCAAAGGGTGGTTCGTTCTC-3'; ptf1a_Cre_Fw 5'-ATAGGCTACCTGGCCATGCCC-3'; ptf1a_ Cre_Rv 5'-CGGGCTGCAGGAATTCGTCG-3').

\section{CRISPR sgRNA design}

CRISPR sgRNAs for Kras, Trp53 and Lkbl have been previously described [19]. Genomic sequence for Aridla gene was downloaded from UCSC genome browser and sgRNA cassette was generated using the CRISPR design tool (http://crispr.mit.edu).

\section{AAV vector plasmids}

The AAV-KPL plasmid (AAV: ITR-U6-sgRNA (Kras)-U6sgRNA (p53)-U6-sgRNA (LKB1)-pEFS-RLUC-2A-CreshortPA- KrasG12D_HDRDonor-ITR) was obtained from Addgene. AAV-KPL $\Delta$ Cre was generated by removing the Cre sequence from AAV-KPL by digesting with Nhe1 and HindIII followed by ligation. AAV-KP $\Delta$ Cre was generated by digesting AAV-KPL $\Delta$ Cre with Kpn1 and Xba1 and followed by ligation. AAV-K $\Delta$ Cre was made by digesting AAV-KPL $\Delta$ Cre with BamH1 and Kpn1, and followed by ligation. To construct AAV-KPA $\Delta$ Cre, first the sgRNA for mouse Aridla exon3 was cloned into the pX459 (pSpCas9 (BB)-2A-Puro) vector, then the whole segment containing the U6 promoter, Aridla sgRNA with primers containing Xba1 and Kpn1 sites was PCR amplified. Finally, AAV-KPL $\Delta$ Cre was digested with Xba1 and Kpn1 to release the $L k b 1$ CRISPR containing unit following which the entire amplicon containing Aridla CRISPR was ligated to generate AAV-KPA $\Delta$ Cre. AAV-KA $\Delta$ Cre was made by digesting AAV-KPA $\Delta$ Cre with BamH1 and Xba1 and re-ligated. All of the AAV vector constructs were sequence verified before virus production. All vector constructs are readily available from the authors upon request (the schematic of the constructs are shown in Supplementary Fig. 1A).

\section{AAV vector production}

Human Embryonic Kidney 293 T (HEK293T) cells were used for AAV production. HEK293T cells were maintained in Dulbecco's Modified Eagle Medium (DMEM, Life Technologies) supplemented with $10 \%$ fetal bovine serum (FBS, Atlanta-Biologicals) and $1 \%$ penicillin and streptomycin (Life Technologies). Cells were grown as adherent cultures in $5 \% \mathrm{CO} 2$ at $37^{\circ} \mathrm{C}$ on $15 \mathrm{~cm}$ cell culture plates. Viruses were generated by linear polyethyleneimine (PEI)mediated triple transfection. The PEI was added to a DNA mixture of $\mathrm{pAAV} 2 / 8$, pAAV2/5, or $\mathrm{pAAV} 2 / 6$ rep/cap plasmid $(10 \mu \mathrm{g} / \mathrm{plate})$, transgene plasmid (10 $\mu \mathrm{g} / \mathrm{plate})$, and pXX6-80 helper plasmid (20 $\mathrm{\mu g} /$ plate), incubated for $30 \mathrm{~min}$ at room temperature and added to poly-L-lysine-coated plates of $90 \%$ confluent HEK293T cells. The cells were harvested $48 \mathrm{~h}$ post transfection and lysed by three cycles of freeze/thaw. The cell lysate was treated with $4 \mathrm{U} / \mathrm{ml}$ Benzonase (Sigma-Aldrich) for $40 \mathrm{~min}$ at $37^{\circ} \mathrm{C}$, and then separated through an iodixanol gradient $(15 / 25 / 40 / 54 \%)$ in Beckman Ultra-Clear Quick Seal Tubes (Beckman Coulter, Brea, CA). After centrifugation at 48,000 rpm for $1.75 \mathrm{~h}$ in a $70 \mathrm{Ti}$ rotor, the virus was extracted from the $40 \%$ layer. Viruses were further purified through anion exchange chromatography (Q Sepharose, Pall) followed by concentration and buffer exchange through Amicon Ultra 4 (Millipore) into GB-PF68 (50 mM Tris, pH 7.6, $150 \mathrm{mM}$ $\mathrm{NaCl}, 10 \mathrm{mM} \mathrm{MgCl} 2,0.001 \%$ Pluronic F68). Virus titers were determined by qPCR using SYBR green (Life Technologies) and primers against the Rluc portion of the transgene cassette (forward: GCCTCGTGAAATCCCGT TAGTA, reverse: GCATTGGAAAAGAATCCTGGGT CC) on a BIO-RAD CFX96.

\section{Surgical procedure and direct injection}

A volume of $100 \mu \mathrm{l}$ of PBS containing AAV-CRISPR sequences $\left(10^{11}\right.$ viral genome copies) was injected into the pancreatic tail of 6 weeks old Rosa p48-Cre;LSL-Cas 9 mice using $29 \mathrm{G}$ needle (BD Biosciences) through left subcostal laparotomy under inhaled anesthesia. Leakage was not observed in any mice during the procedure. After the injection, the pancreas and spleen was carefully returned into the peritoneal cavity and the abdomen was closed using 4-0 Vicryl (Ethicon) and skin staplers. All the mice were monitored at least once a week by abdominal palpation and abdominal ultrasound to investigate the entire pancreas was performed every 4 weeks using the Vevo 2100 (Fujifilm). Moribund mice with pancreatic tumors were euthanized by cervical dislocation and carbon dioxide inhalation according to the IACUC protocol. The assessment of primary tumor, intraperitoneal dissemination and distant metastases performed were based on both gross findings at necropsy and by histopathological examination. Any surviving mice at 100 days ( $\sim 3$ months post injection) were then euthanized and the Pancreata harvested for histopathological examination.

\section{T7 endonuclease gene editing assay}

Genomic DNA was isolated from tumor samples using the Qiagen genomic DNA isolation kit. Subsequently 400 bp of genomic fragment encompassing the CRISPR targeted cleavage site was PCR amplified with high fidelity Taq polymerase. Approximately $200 \mathrm{ng}$ of purified PCR product was used for each T7 endonuclease assay. Briefly, $2 \mu$ NEBuffer 2, $200 \mathrm{ng}$ of purified PCR product and $\mathrm{dH} 2 \mathrm{O}$ was added to a total of $19 \mu \mathrm{l}$ in a PCR tube. The hybridization reaction was 
run as follows: $5 \mathrm{~min}, 95^{\circ} \mathrm{C}$; ramp down to $85^{\circ} \mathrm{C}$ at $-2{ }^{\circ} \mathrm{C} / \mathrm{s}$; ramp down to $25^{\circ} \mathrm{C}$ at $-0.1{ }^{\circ} \mathrm{C} / \mathrm{s}$; hold at $4{ }^{\circ} \mathrm{C}$. We then added $1 \mu \mathrm{l}(10 \mathrm{U}) \mathrm{T} 7$ endo I and incubated at $37^{\circ} \mathrm{C}$ for $15 \mathrm{~min}$. The reaction was stopped by adding $2 \mu \mathrm{l}$ of $0.25 \mathrm{M}$ EDTA and loaded immediately on a $1.5 \%$ agarose gel.

\section{Immunofluorescence and immunohistochemistry}

Pancreata were harvested from the moribund mice due to tumor burden at different time points, with maximum harvesting time around $\sim 100$ days. Pancreata were fixed in $4 \%$ paraformaldehyde, followed by standard paraffin embedding. Tissue was then cut into $5 \mu \mathrm{M}$ sections. Incubations with primary antibodies were performed overnight at $4{ }^{\circ} \mathrm{C}$ using standard techniques in PBS containing $0.2 \%$ Triton and $10 \%$ FBS using the following antibodies or probes: mouse anti-ECadherin (BD Transduction 610181, 1:50); rabbit anti-Sox9 (Millipore AB5535, 1:1000); hamster anti-Muc1 (Thermo Fisher HM-1630-F, 1:100); mouse anti-Arid1a (Santa Cruz Biotechnology sc-32761, 1:100); mouse anti-p53 (Cell Signaling Technology \# 2524) and rabbit anti-LKB1 (Abcam ab185734 1:100). For immunofluorescence, secondary antibodies were obtained from Abcam and used at 1:300 dilution; samples were mounted with Fluorescence mounting media (Dako, S3023) and imaged on the Olympus Confocal Microscope. For immunohistochemistry, EnVision HRP secondary antibodies (Dako K4001) were imaged on an Olympus BX43 microscope.

\section{Whole-exome sequencing}

Whole exome sequencing of harvested murine tumors was performed at Johns Hopkins University Next Generation Sequencing (NGS) Core Facility. Briefly, the Agilent SureSelect Mouse exome sequencing kit was used to prepare sequencing libraries and capture annotated exonic sequences. The resulting libraries were sequenced on a HiSeq 2500 , and after quality control checks the fastq files were aligned to genome build mm10 with bwa mem 0.7.7. Picard tools 1.118 were used to remove duplicate reads and GATK 3.6 was used for base recalibration. To call somatic variants, a reference sequence panel of normals was first constructed from C57BL mouse strains, and Mutect2 was used with this panel. Annotation was done with snpEFF and snpSIFT.

\section{Results}

\section{Strategy for virally delivered CRISPR-mediated genome editing in the adult pancreatic epithelium}

As a prelude for in vivo gene editing studies we first performed direct injection of AAV containing reporter alleles into the mouse pancreas. To test the differential efficacy of distinct AAV serotypes infecting pancreatic epithelial cell, we injected AAV6-Cre-GFP and AAV8-Cre-GFP into the adult mouse Pancreata (Supplementary Figure. 1B, C) which confirmed the superiority of AAV8 in infecting epithelial cells as evidenced by extent of GFP expression. When calculated, AAV8 serotype yielded more than 12 times higher number of cellular transduction based on GFP expression, when compared with AAV6 (Supplementary Figure. 1D). Henceforth, all of our AAV vectors were made using AAV8. Further, in order to avoid spurious $\mathrm{Cre}$ dependent recombination in the non-epithelial pancreatic tissue including the peritoneum from the injected virus, we deleted the Cre sequence from the original AAV construct, AAV-KPL (Supplementary Figure. 1A). Instead, we generated previously described $p 48-\mathrm{Cr}^{+/} ; \mathrm{LSL}-\mathrm{Cas} 9$ mice as the "workhorse" mouse where Cre recombinase is only expressed in the adult pancreatic epithelium, specifically acinar cells.

\section{Multiplexed CRISPR/Cas9-mediated somatic genome-editing results in autochthonous PDAC harboring diverse genotypes}

A set of six independent AAV8 vectors were generated for injection into the pancreas of $p 48-\mathrm{Cr}^{+/-} ; \mathrm{LSL}-\operatorname{Cas} 9$ mice, containing the following sgRNAs: (i) $A A V-L a c Z$ (control) (ii) $A A V$-Kras (which included the Kras ${ }^{G 12 D}$ HDR template and henceforth designated as "K", (iii) AAV-Kras, Trp53 (henceforth designated as "KP"), (iv)AAV-Kras; Aridla (henceforth designated as "KA"), (v) AAV-Kras; Trp53; $L k b 1$ (henceforth designated as "KPL") and (vi) $A A V$-Kras; Trp53;Aridla (henceforth designated as "KPA"). The schematic of the model development and post-harvest correlative assays are illustrated in Fig. 1a. The pancreatic development was monitored in real time by ultrasound as shown in Fig. 1b. The survival of the mice injected with the six vectors is depicted in Fig. 1c. Excluding the control "LacZ" mice, the mice with "K" and "KA" had the most indolent natural history with no mortality observed at $\sim 100$ days (at which point these mice were euthanized). In contrast, "KP", "KPA" and "KPL" injected mice had comparable median survival with progression to invasive neoplasia. In the "KPL" injected nice, ultrasound examination demonstrated readily visible tumors as early as eight weeks after injection and these were apparent 1-2 weeks later in "KP" and "KPA" injected mice.

Representative histopathology photomicrographs from the mice injected with various AAV constructs are shown in Fig. 2. In mice that succumbed to invasive carcinomas, we have captured examples of murine pancreatic intraepithelial neoplasia (mPanIN) in the surrounding pancreas, to demonstrate that the carcinomas arose on a backdrop of 


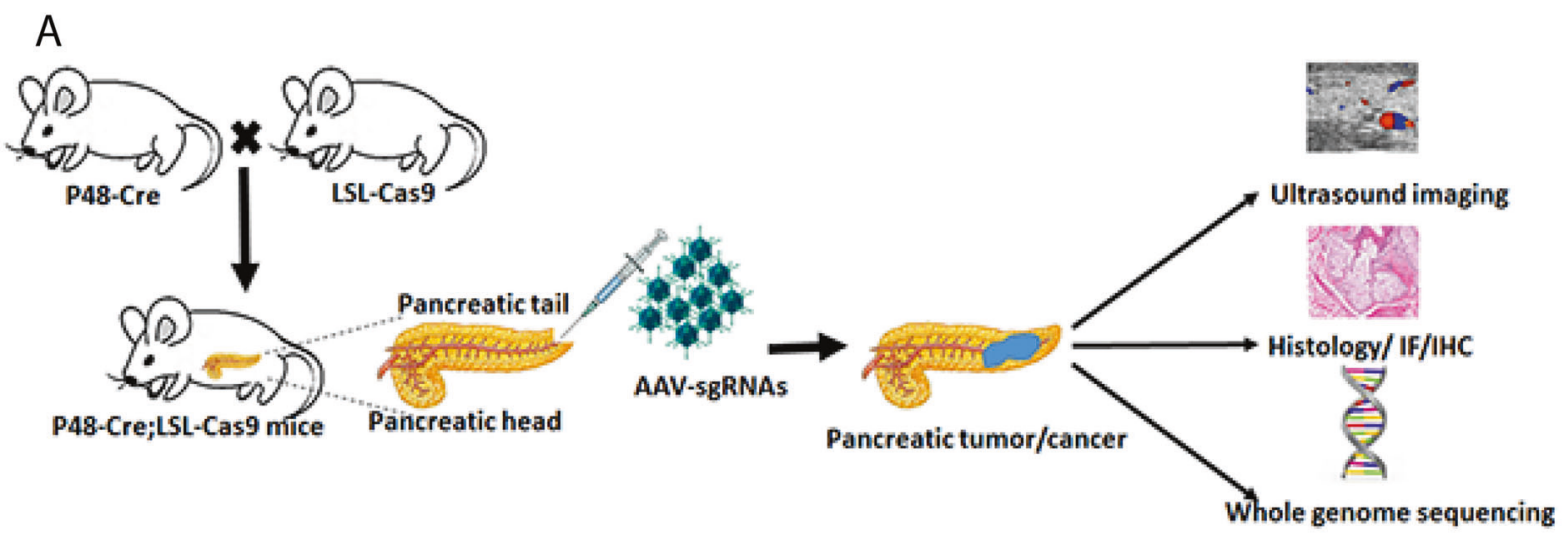

B

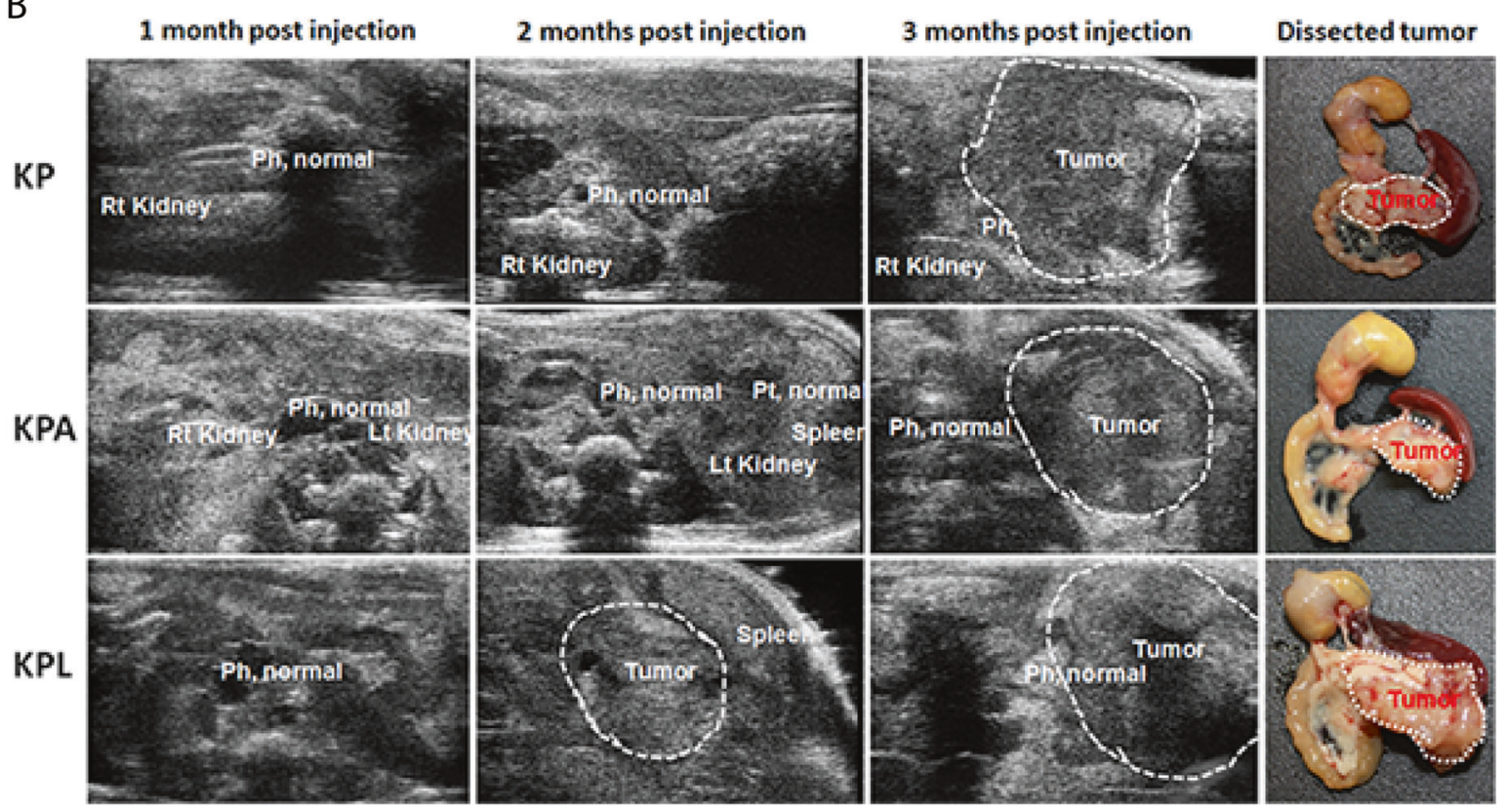

C

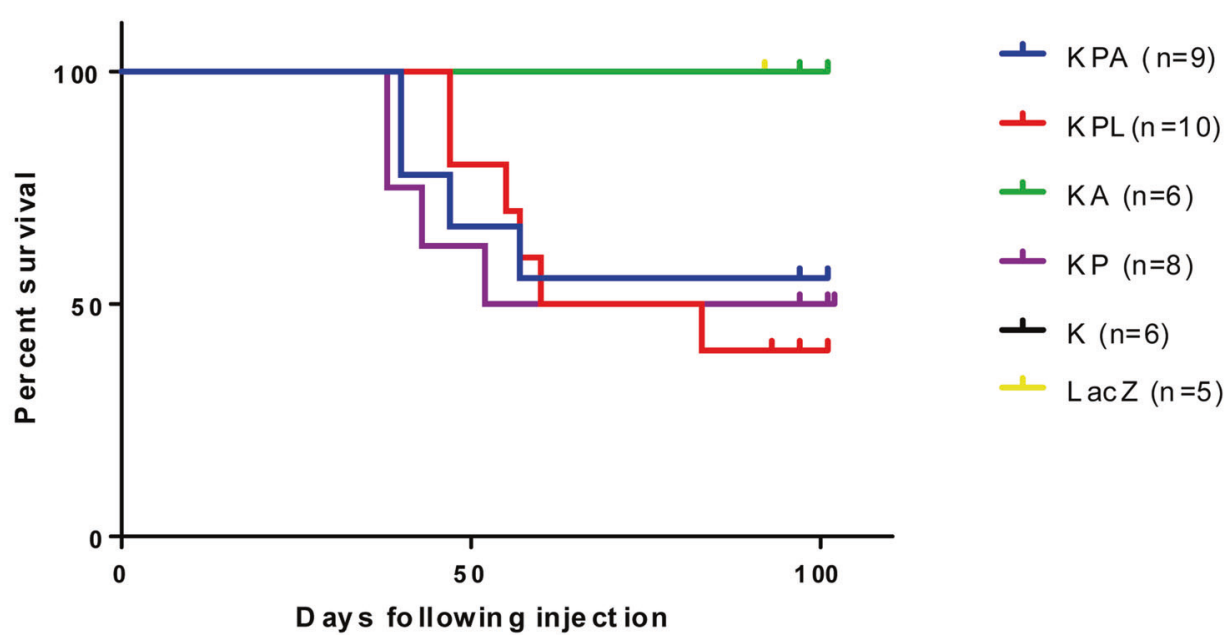

what is widely accepted as the most common precursor lesion in human PDAC. In "K" and "KA" injected mice, we only observed focal mPanIN lesions of various grades, in the absence of invasive carcinomas (Fig. 2a-d), which is also reflected in their survival. Notably, surrounding histologically normal pancreatic parenchyma was readily seen in 
Fig. 1 Generation and natural history of CRISR/Cas9 induced genetically engineered mouse models of pancreatic cancer. a Schematic of experimental procedure showing the direct injection of AAVs containing sgRNAs into the tail of $p 48$-Cre; LSL-Cas 9 mice. Serial ultrasound imaging was performed to follow tumor induction for $\sim 3$ months following injection, at which time point the mice were euthanized and the pancreata were harvested for histopathological evaluation and correlative studies, b Non-invasive monitoring of pancreatic tumor formation by ultrasound imaging at regular interval following viral injection. Note the normal pancreas head $(\mathrm{Ph})$ at 3 months post injection while the pancreatic tail harbors a large tumor. Relative position of the pancreas with respect to kidney and spleen are depicted on the ultrasound image. c The natural history of various tumor genotypes generated in this study - LacZ (control), "K", "KA", "KP", "KPA", and "KPL" is show via Kaplan-Meier survival curve. Any remaining mice alive at 100 days post injection were euthanized and underwent necropsy. Please see text for details of each genotype

these mice even at $\sim 100$ days, distinct from the pancreaswide activation of $\mathrm{Cre}$ in conventional models where there is progressive replacement of acinar tissue by precursor lesions throughout the organ. In contrast to mice injected with "K" and "KA" viruses, the remaining cohorts developed invasive cancers, as seen with "KP" (Fig. 2f), "KPA" (Fig. 2h) and "KPL" (Fig. 2j) respectively. Of note, we also readily observed examples of acinar ductal metaplasia (ADM) in the background parenchyma and typically adjacent to mPanINs, as these are considered the "precursor to the precursor" in the multistep model of murine PDAC pathogenesis (Fig. 2e, g, i). Further, the "KPL" injected mice were distinguished by the presence of cystic precursor lesions (Intraductal papillary mucinous neoplasms or IPMNs, illustrated in Supplementary Figure 2), a feature that has been previously reported in conventional Cre models of $L k b 1$ deletion. The resulting IPMNs expressed the apomucin MUC1 on their surface, consistent with a socalled pancreato-biliary subtype of differentiation (Supplementary Figure 3).

Histopathological examination of the invasive carcinomas at low magnification often showed normal pancreatic parenchyma adjacent to a region of invasive carcinoma mirroring the "segmental" nature of disease in the human pancreas (and distinct from the multifocal nature of disease seen in the conventional Cre models) (Supplementary Figure 4A). While adenocarcinomas were seen with all three cancer forming genotypes ("KP", "KPA" and "KPL") (Fig. 2), the proportion of adenocarcinomas versus poorly differentiated (sarcomatoid) tumors varied (Supplementary Figure 4B), with "KPL" and "KPA" resulting predominantly in adenocarcinomas and "KP" in poorly differentiated lesions. These variations in the proportion of histological subtypes for individual genotypes have been well documented with $\mathrm{Cre}$ - based models [34], so was not unexpected. Quantification on the basis of surface area occupied by low-grade and high-grade PanIN lesions, and

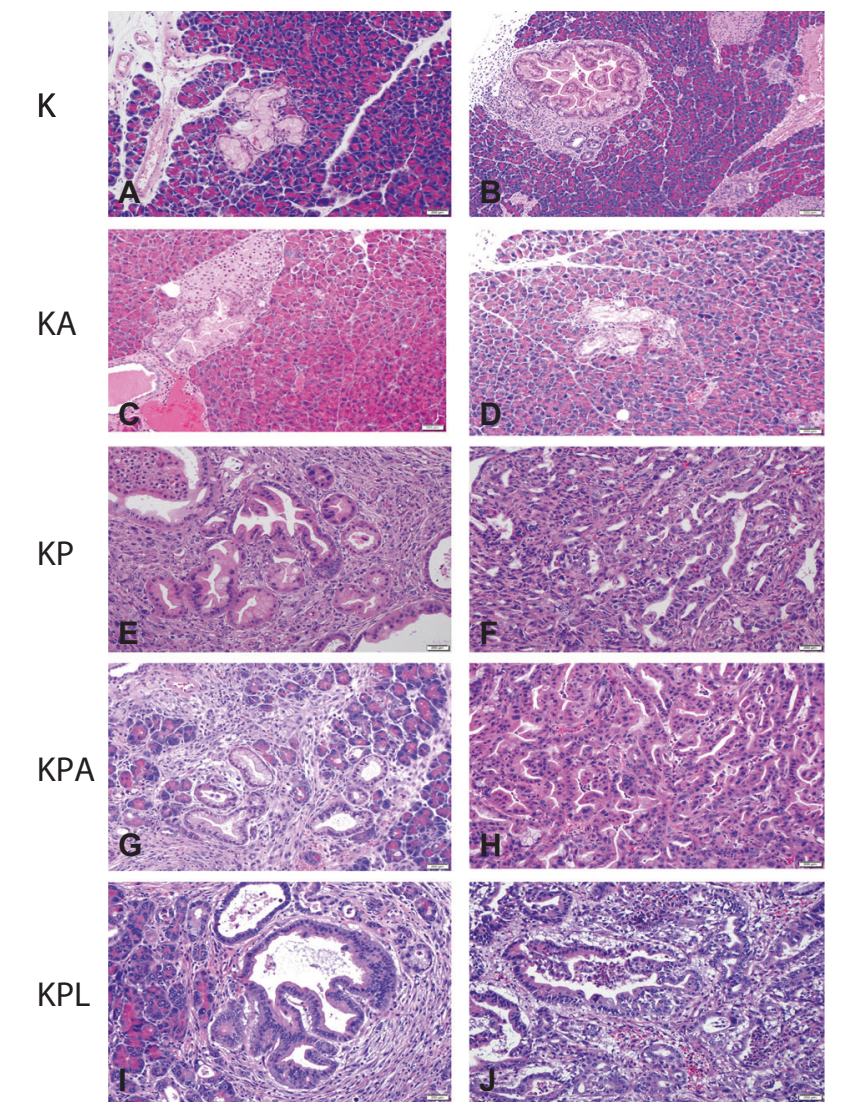

Fig. 2 Histopathology of CRISPR-Cas9-induced pancreatic precursor lesions and invasive cancers in $p 48$-Cre; LSL-Cas 9 mice. a Low-grade murine pancreatic intraepithelial neoplasia (mPanIN) lesion on an otherwise uninvolved pancreatic parenchyma in an AAV-Kras ("K") mouse. No invasive cancers were seen at $\sim 100$ days. b High-grade murine mPanIN lesion on an otherwise uninvolved pancreatic parenchyma in an AAV-Kras ("K") mouse. No invasive cancers were seen at $~ 100$ days. c, d Low-grade murine mPanIN lesion on an otherwise uninvolved pancreatic parenchyma in AAV-Kras; Aridla ("KA") mice. No high-grade $\mathrm{mPanIN}$ or invasive cancers were seen at $\sim 100$ days. e Entrapped high-grade murine mPanIN lesions with poorly differentiated (sarcomatoid) PDAC in the background in an AAV-Kras, Trp53 ("KP”) mouse. f Invasive adenocarcinoma (PDAC) arising in an AAV-Kras, Trp53 ("KP") mouse. g Low-grade murine $\mathrm{mPanIN}$ lesions and acinar ductal metaplasia (ADM) in a pancreas with changes of chronic pancreatitis in an AAV-Kras, Trp53, Arid1A ("KPA") mouse. $\mathbf{h}$ Invasive adenocarcinoma (PDAC) arising in an AAV-Kras, Trp53, Arid1A ("KPA") mouse. i High-grade murine mPanIN lesions and acinar ductal metaplasia (ADM) in a pancreas with changes of chronic pancreatitis in an AAV-Kras, Trp53, Lkb1 ("KPL") mouse. j Invasive adenocarcinoma (PDAC) arising in an AAV-Kras, Trp53, Lkb1 ("KPL") mouse

invasive carcinoma (poorly differentiated and ductal adenocarcinoma), are shown in Supplementary figure 4C. High-grade PanINs were seen in the 3-month cohorts of "KPA" and "KPL" mice, respectively, while "K" and "KA" exhibited only low-grade lesions, even at 3-months of age. In addition to the primary tumor, metastatic adenocarcinomas were observed in a subset of "KPL"-injected mice, 
specifically in the liver (10\% of the injected mice) and lung (20\% of the injected mice) (Supplementary Figure 5).

\section{Correlative studies in CRISPR/Cas9 generated PDAC models}

A series of correlative protein expression and sequencing studies were conducted on the harvested tissues from the CRISPR/Cas9 generated PDAC lesions. For example, we confirmed the loss of expression of Arid1a and Lkb1 within the neoplastic cells of tumors harvested from "KPA" and "KPL" virus-injected pancreata, respectively (Fig. 3, upper panel). Similarly, loss of nuclear p53 protein was confirmed by immunohistochemistry in the PanIN region of the "KP" virus injected mouse when compared to 6-month-old $p 48$ cre; LSL-Kras control mouse (KC) where p53 proteins are highly expressed in the nuclei of the PanIN region (Fig. 3, lower panel). Although "KA" injected mice did not form invasive carcinomas, partial to complete loss of expression of Arid1a protein was demonstrable in the ADM and $\mathrm{mPanIN}$ lesions that resulted in the pancreata (identified based on Sox9 expression) consistent with successful somatic editing of Aridla loci (Supplementary Figure 6). As previously noted "KP" mice developed multiple examples of poorly differentiated (sarcomatoid) carcinomas, and these poorly differentiated PDAC were characterized by loss of membrane E-cadherin and co expression of the mesenchymal marker vimentin, suggesting a phenotype of epithelial to mesenchymal transformation (EMT), which has been previously described in aggressive PDAC [35, 36].

The $\mathrm{T} 7$ endonuclease assay serves as a preliminary measure of the efficacy of genome targeting based on the ability of the enzyme to cleave DNA strands that are not perfectly matched due to non-homologous end joining. Representative examples of the T7 endonuclease assay for Kras, Trp53 and Lkb1 from "KPL" and "KPA" tumors are shown in Fig. 4a, which demonstrates targeting of sgRNA to the corresponding locus. In contrast to the cancer samples, the control pancreas injected with LacZ sgRNA showed no evidence of genome editing in the T7 endonuclease assay.

In order to confirm the CRISPR/Cas9-mediated genome editing at the nucleotide level, we then performed exome sequencing of tumor-derived genomic DNA from "KPL" and "KPA" virus injected pancreata, using reference DNA from a panel of C57/BL mice. In Fig. 4b, we demonstrate NGS reads for KRAS, showing replacement of the endogenous locus with the donor template carrying the codon 12 non-synonymous mutation by HDR. Additional "marker" nucleotides (in blue color on sequence read) derived from the exogenous KRAS template (and accounting for synonymous base variations) are seen in the neoplastic DNA, confirming that the $\operatorname{Kras}^{G 12 D}$ mutation observed was not
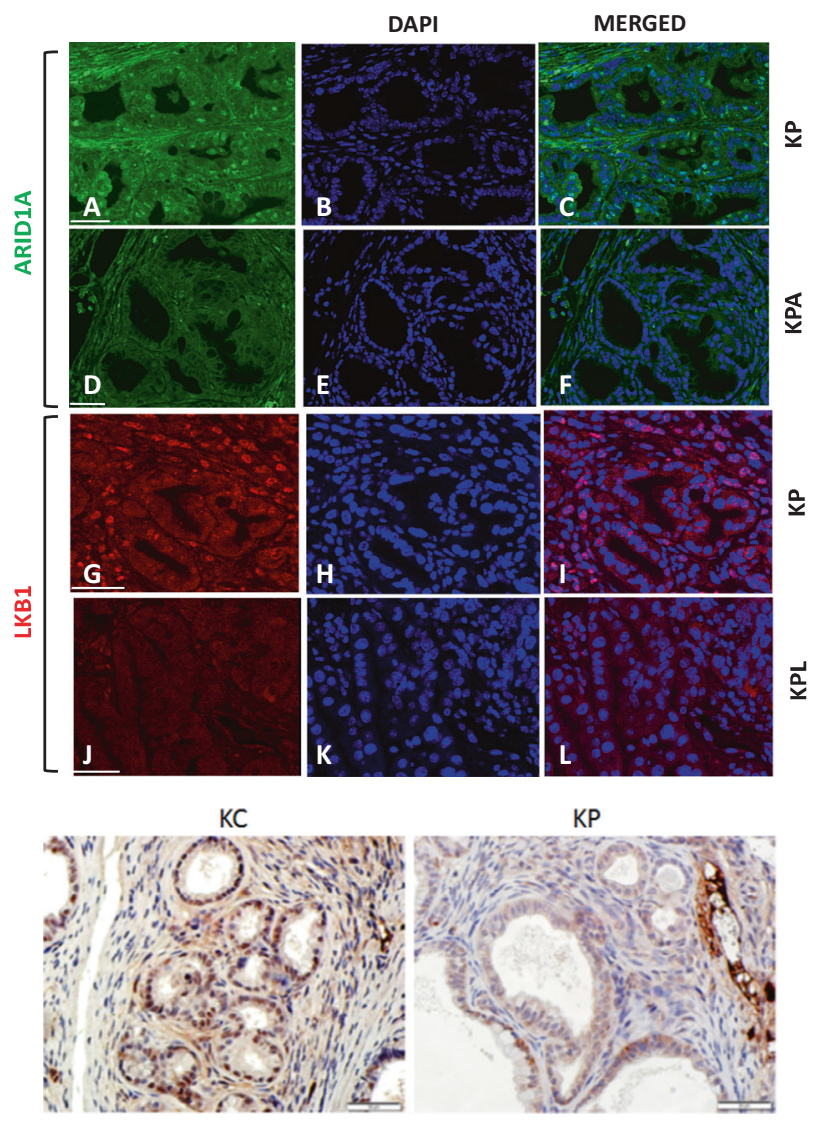

Fig. 3 Downregulation of CRISPR/Cas 9 targeted protein in PDAC samples. Upper panel: a-f Immunofluorescence for Aridla shows loss of nuclear Aridla expression in "KPA" tumors, with retained expression in "KP" tumors. g-i Immunofluorescence for Lkb1 shows loss of nuclear Lkb1 expression in "KPL" tumors, with retained expression in "KP" tumors. In this photomicrograph panel, $\mathbf{a}$ and $\mathbf{d}$ are Arid1a, $\mathbf{g}$ and $\mathbf{j}$ are LKB1 stains, respectively. $\mathbf{b}, \mathbf{e}, \mathbf{h}$, and $\mathbf{k}$ are DAPI stains. The scale bar $=50 \mu \mathrm{m}$. Lower panel: immunohistochemistry showing nuclear p53 staining in the control KC (p48-cre; LSL-Kras) mouse and absence or reduced nuclear p53 in "KP" mouse. The scale bar $=50 \mu \mathrm{m}$

spontaneous but rather derived from the exogenous template by HDR. Since "bulk" tumor DNA was sequenced, we observed a mutant fraction of $9 \%$ for the $\mathrm{Kras}^{G 12 D}$ allele. Exome sequencing data also confirmed disruption of three loci (Aridla, Trp53 and Lkb1), with multiple insertion deletion ("indel") events observed for each gene (Fig. 4c-e). This is consistent with independent CRISPR editing events occurring during the initial injection, and the observed PDAC "mass" at necropsy being of oligoclonal origin. The pie charts adjacent to each gene designate an overview of the classes of indel events for each targeted locus (detailed information for individual mouse tumors sequenced is illustrated in Supplementary Figure 7). The majority of indels were located $1-5 \mathrm{bp}$ upstream or downstream of the PAM sequence. The maximum insertion size observed was $15 \mathrm{bp}$ and the maximum deletion size observed was $23 \mathrm{bp}$. 
A

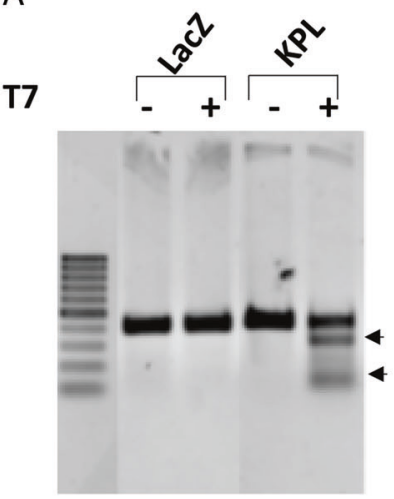

Kras

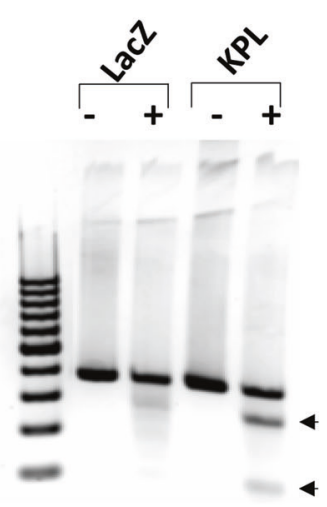

p53

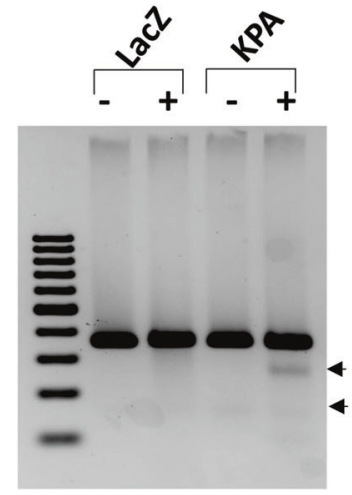

ARID1A

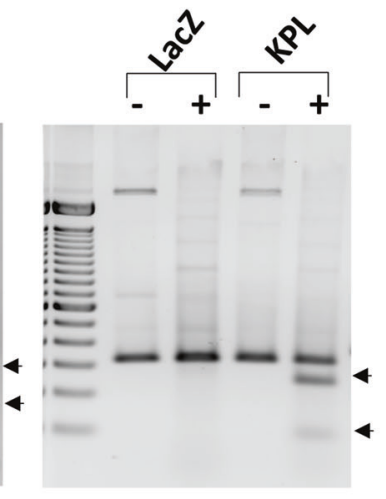

LKB1

B

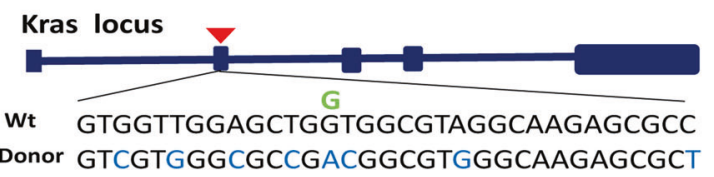

r1 GTCGTGGGCGCCGACGGCGTGGGCAAGAGCGCT

r2 GTCGTGGGCGCCGACGGCGTGGGCAAGAGCGCT

r3 GTCGTGGGCGCCGACGGCGTGGGCAAGAGCGCT

r4 GTCGTGGGCGCCGACGGCGTGGGCAAGAGCGCT

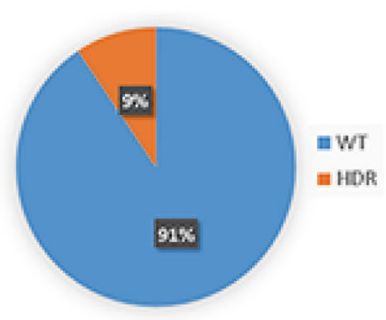

C
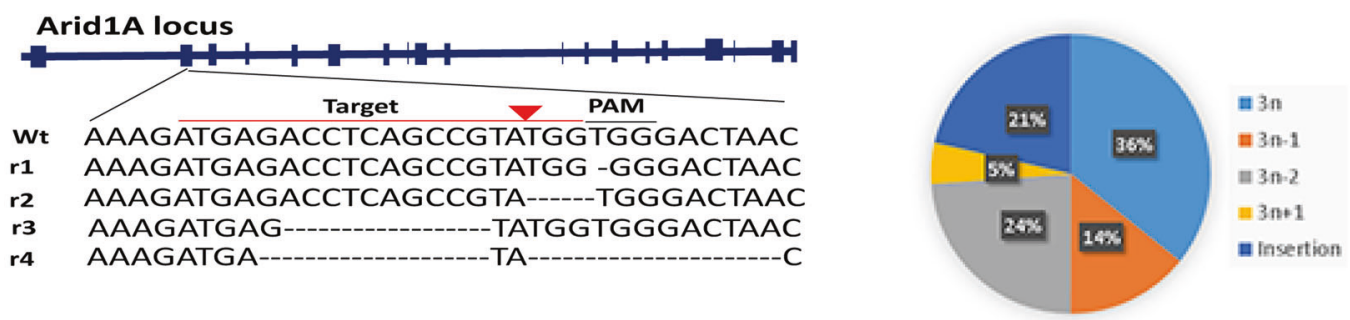

D

\section{p53 locus}
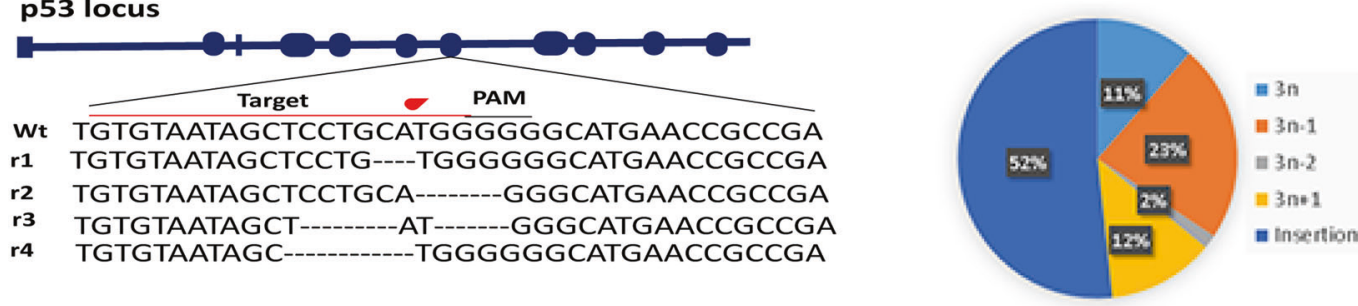

$\mathrm{E}$
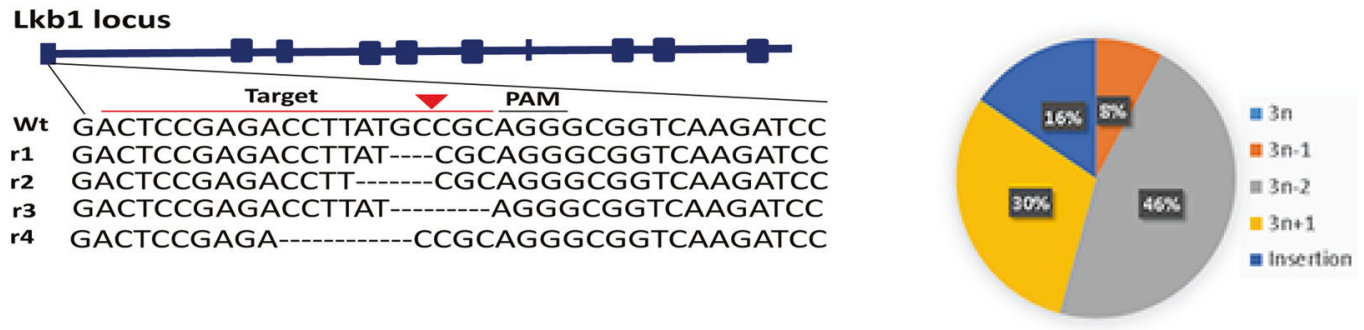

Overall, between 8 and $30 \%$ of DNA reads (based on underlying neoplastic cellularity and gene of interest) demonstrated unequivocal indels on sequencing.
Finally, we explored whether the resulting tumors had developed secondary mutations of the 12 most commonly altered genes in PDAC, using data collated from the TCGA 
Fig. 4 Sequencing of CRISPR/Cas9-induced tumors confirms homology-directed recombination (HDR) and gene editing at targeted loci. a T7 endonuclease assay in genomic DNA isolated from "KPL" and "KPA" tumors following injection of AAV-sgRNAs constructs demonstrates evidence of gene targeting at the corresponding loci. Genomic DNA from AAV-LacZ injected pancreas was used as control. "-" and "+" indicates absence or presence of T7 endonuclease, respectively. b Schematic of sgRNA position and HDR donor template design for targeting the mouse endogenous Kras locus to incorporate a $\operatorname{Kras}^{\mathrm{G} 12 \mathrm{D}}$ oncogenic mutation. Next-generation sequencing (NGS) reads confirms a G-A point mutation (box) leading to $\operatorname{Kras}^{\mathrm{G} 12 \mathrm{D}}$ while blue letters represent exogenous bases from the introduced HDR template that result in synonymous changes. The pie chart on the right represents the percentage of HDR at the Kras locus from the total number of NGS reads across all six of the sequenced mouse tumors. c-e Schematic representing the sgRNA design for targeting Aridla, Trp53 and $L k b 1$, respectively, and representative NGS reads $\left(r_{\mathrm{n}}\right)$ confirming the presence of "indels" that disrupt the target of interest (left). Notably, any given tumor demonstrated multiple indels at the targeted locus, consistent with a oligoclonal nature of the resulting neoplasm. The distribution of indel classes at each target site of interest is shown in the pie charts on the right. $3 n$ represents in frame mutations while $3 n-1,3 n-2$ and $3 n+1$ represent different out of frame mutations

and ICGC datasets [3, 4]. Besides the CRISPR targeted genes in the corresponding mouse model (Supplementary Figure 8), we did not observe any secondary mutations in genes such as Smad4, Gnas, Rnf43, Kdm6a and Brca2. Importantly, the notable exceptions were Kmt2c (Mll3) and $K m t 2 d$ (Mll4), which encode for chromatin regulatory proteins [37], and demonstrated frameshift mutations or "indels" in subsets of tumors. It is unclear whether these events represented a requirement for tumor progression in these models, given that in the TCGA and ICGC datasets, genes encoding for chromatin regulatory proteins are one of the most commonly mutated family of genes $[3,4]$.

\section{Discussion}

GEMMs represent the most rigorous preclinical platform for hypothesis testing the functional contribution of recurrent genetic alterations towards multistep pancreatic carcinogenesis, but conventional approaches demonstrate several logistical and temporal challenges, particularly, when complex allelotypes are desired. In this study, we have successfully demonstrated CRISPR-induced somatic gene editing using AAV-mediated direct intra-pancreatic injection of diverse sgRNA combinations for establishing autochthonous PDAC with high efficiency, and for some of the genotypes, relatively short latency to cancer incidence. Specifically, while five distinct sgRNA combinations were used as AAV cargo and all developed mPanINs, three genotypes ("KP", "KPA", and "KPL") resulted in PDAC in the arbitrarily assigned time frame for observation ( $\sim 3$ months), underscoring the importance of the genetic background on the latency of cancer formation. While it is certainly possible that the remaining two genotypes (" $\mathrm{K}$ " and "KA") might eventually develop PDAC on longer follow up, our goal in this initial feasibility study was to keep the time frame for observation relatively short and perform necropsy on any surviving mice at $\sim 3$ months. Importantly, all of the resulting PDAC lesions developed on a backdrop of credentialed precursor lesions (mPanINs and IPMNs), and progressed to metastatic disease in a subset of mice, confirming that the autochthonous models we have developed recapitulates the multistep progression seen in conventional Cre-based models. Loss of CRISPR-disrupted protein expression was confirmed in both pre-invasive and invasive neoplastic lesions, and NGS on the resulting tumors validated gene targeting at the sequence level.

The catalogue of genetic alterations targeted using CRISPR sgRNAs in the pancreas were based on the frequency of alterations reported in human PDAC in public databases [3, 4]. For example, KRAS, which is activated by oncogenic mutations in $>90 \%$ of PDAC, and TP53, which is inactivated by loss of function mutations of homozygous deletions in $75 \%$ of PDAC, are obvious choices. In fact, mutations of KRAS are required not only for the cell autonomous effects in cancer progression, but also for the paracrine effects on the tumor microenvironment that create a tumor permissive immune milieu [38, 39]. Somatic mutations of the SWI/SNF complex ARIDIA are seen in approximately $8-10 \%$ of PDAC, and this DNA binding helicase has been implicated in maintenance of acinar cell identity, and attenuating the progression to a precursor ductal phenotype (acinar ductal metaplasia) on a mutant Kras background [40, 41]. In our series, confirmed loss of Aridla and expression of mutant Kras in "KA" mice was sufficient to induce mPanINs, but not PDAC within the $\sim 3$ month time window. In contrast, addition of Trp53 deletion ("KPA") resulted in PDAC with relatively short latencies, with a greater propensity towards differentiated adenocarcinomas than the poorly differentiated carcinomas observed in "KP" mice alone. Of note, this correlation between ARIDIA mutations and histological grade of differentiation has also been reported in other solid cancers, such as endometrial, hepatocellular, and pancreatic carcinomas [42-44], suggesting that loss of this epigenetic regulator might promote the acquisition of a ductal (i.e. "differentiated") phenotype in PDAC. Finally, we also targeted Lkb1, which encodes for a serine threonine kinase mutated in several solid cancers. In the pancreas, LKBI mutations or loss of Lkb1 expression has been described in cystic precursors (IPMNs) [45, 46], and prior conventional models of conditional $L k b l$ loss have demonstrated PDAC arising on the background of cystic neoplasms [47, 48]. In our series, we did not examine "KL" as a dual targeting construct, but "KPL" injected mice developed cystic lesions 
resembling IPMNs in a subset of cases. Similar to "KPA" mice, "KPL" mice also developed predominantly adenocarcinomas upon progression.

In our study, CRISPR-mediated expression of mutant $\operatorname{Kras}^{G 12 D}$ from the endogenous locus in the p48-expressing mature acinar compartment resulted in the spontaneous induction of entire spectrum of mPanIN and PDAC in the "KP", "KPA" and "KPL" cohorts, without the need for concomitant pancreatitis (such as cerulein-induced chemical pancreatitis). This confirms multiple previous reports, including our own work, supporting the ability of mature acinar cells to undergo neoplastic transformation upon expression of mutant Kras under the endogenous promoter [49-51] without the requirement for exocrine injury. This is in contrast to a prior study that stated the absolute requirement for inflammation to induce neoplastic lesions in mature acinar cells [52], which we believe may have been a result of the 3'-modified mutant Kras allele used in that report. We do not believe that the accelerated mPanIN and PDAC formation in the current dataset is a result of pancreatitis induced by AAV injection into pancreas, as similarly injected "KA" and "K" demonstrated focal mPanINs only, underscoring the importance of the altered genotype within targeted acinar cells.

A few important methodological caveats emerged during the course of this study. First, we recognized the AAV8 serotype to be significantly more efficient at viral transduction than AAV6, which is consistent with prior reports of using AAV8 as a delivery vector to the pancreas [53]. Second, the original CRISPR construct used in this study had a Cre expressing element [19], which resulted in spurious $\operatorname{Cas} 9$ activation and extra-pancreatic mesenchymal tumors in the abdomen due to leakage of injected virus (data not shown), therefore, we deleted the Cre element, thus restricting recombinase expression to only the p48expressing acinar parenchyma in the p48-Cre; LSL-Cas9 mouse. Third, while there have been prior studies using CRISPR/Cas 9 for somatic gene editing and PDAC induction in mice, there is one key distinction from our approach. For example, Chiou et al. [26] used an intraductal lentiviral injection methodology for delivering Cre recombinase and sgRNA against $L \mathrm{kbl}$ into the main pancreatic duct of mice expressing conditional ("LSL") allele of Cas9 and mutant Kras $^{\text {G12D }}$. Analogously, Maresch et al. [27] used direct electroporation of plasmid based CRISPR/cas9 vectors against as many as 13 targeted loci in Ptfl-Cre; LSLKras $^{\mathrm{G} 12 \mathrm{D}}$ mice, resulting in invasive tumors with multiple disrupted alleles. Both of these methods, however, required a conditional mutant LSL-Kras ${ }^{\mathrm{G} 12 \mathrm{D}}$ allele in the host animal (either constitutively expressed [27], or activated via virally delivered Cre [26]), on which background, somatic gene editing was enabled. In our study, however, we have dispensed with the requirement for a $\operatorname{Kras}^{\mathrm{G} 12 \mathrm{D}}$ allele in the host animal, by incorporation of a mutant template sequence in the AAV vector that is incorporated at the endogenous Kras locus through HDR. This has allowed us to utilize a single uniform strain of "workhorse" mice - Ptfl-Cre; LSL-Cas 9 for injecting various sgRNAs, thereby, greatly enhancing the efficiency of PDAC generation when using different combinations of targeting vectors. Further, by expressing mutant Kras only in the subset of cells where the cooperating tumor suppressor allele is also disrupted, we ensure a bona fide "segmental" nature of disease in the pancreas, recapitulating the cognate human pathophysiology. A potential corollary advantage of this method is the possibility that genes of interest can be independently disrupted, including prior to the activation of mutant Kras, by varying the timing of AAV/CRISPR delivery (for example, with sequential injections).

Of interest, NGS studies on the CRISPR-induced PDAC showed that editing events were typically localized within a few bases of the PAM sequence, with individual "bulk" tumors harboring multiple classes of "indels" or frameshift mutations. This is consistent with the CRISPR-induced PDAC being an oligoclonal mix of more than one initiating clonal event, as previously reported [26]. While we did not pursue this particular line of investigation, one can envision that barcoding individual AAV constructs could allow for rigorous interrogation of selectivity in tumor formation and metastatic ability of distinct genomic alterations even at one given locus. The NGS data also revealed a somewhat robust rate of HDR for Kras in the sequenced "bulk" tumors (9\% on average across six tumors), which is substantially greater than the $1.8 \%$ Kras HDR frequency reported in generating lung cancer models using AAV-mediated delivery of CRISPR [19]. The precise reason for this in unclear, but might represent a higher propensity for HDR in the pancreatic acinar component compared to the pulmonary epithelium, or a greater ability of the AAV8 serotype used in this study to enable HDR in the mouse genome, independent of exonuclease activity, as was recently shown for certain AAV strains [54]. At the same time, we should caution that a $9 \%$ HDR frequency at the Kras locus does not necessarily equate with a $\sim 20 \%$ rate of neoplastic cellularity. In a recent comprehensive study involving both mouse and human PDAC, loss of p53 function inevitably leads to gain in the $\operatorname{Kras}^{G 12 D}$ gene dosage [55], and therefore, an extrapolation to $20 \%$ neoplastic cellularity might be an overestimate. Finally, our sequencing data showed that while several recurrently mutated PDAC-associated genes, including Smad4, Gnas, Rnf43, Kdm6a and Brca2 did not undergo secondary mutations during PDAC development, two genes in particular-Kmt2c (Mll3) and Kmt2d (Mll4), which encode for chromatin regulatory proteins [37]— did demonstrate recurrent alterations. The encoded proteins, Mll3 and Mll4, are histone methyltransferase enzymes that 
are part of a so-called COMPASS-like complex responsible for addition of methyl residues to lysine residues on histones, which function as an "activation mark". Loss of function mutations of $M L L 3$ and other COMPASS-like complex proteins is reported in $\sim 10 \%$ of PDAC, and loss of expression has been associated with better prognosis [37]. We are continuing to explore the relevance of these recurrent non-targeted mutations, including their requirement for tumor formation in the context of other targeted loci using the AAV-CRISPR system.

In summary, we have generated a "workhorse" platform using a previously reported Ptfl-Cre; LSL-Cas 9 mouse that enables the relatively facile interrogation of the functional role of mutated genes in the PDAC landscape, including diverse combinations of targeted alleles using AAV8 as a delivery vector. Our platform precludes the need for a germline mutant Kras allele in the host mouse, and the resulting pancreata demonstrate the full compendium of precursor and invasive lesions observed in human PDAC. This "workhorse" platform will allow investigators to conduct in vivo functional genomics studies and generate reagents, such as cell lines with defined genetic alterations, with considerable efficiency.

Acknowledgements This work was supported by NIH/NCI Grant (5P01CA117969) and NIH/NCI Grant (RO1 CA218004-01A1) awarded to AM. NI and HY were partly supported by post-doctoral fellowship from Uehara Memorial foundation, Japan.

\section{Compliance with ethical standards}

Conflict of interest The authors declare that they have no conflict of interest.

Publisher's note: Springer Nature remains neutral with regard to jurisdictional claims in published maps and institutional affiliations.

\section{References}

1. Ying H, Dey P, Yao W, Kimmelman AC, Draetta GF, Maitra A, et al. Genetics and biology of pancreatic ductal adenocarcinoma. Genes Dev. 2016;30:355-85.

2. Siegel RL, Miller KD, Jemal A. Cancer statistics, 2018. CA Cancer J Clin. 2018;68:7-30.

3. Cancer Genome Atlas Research Network. Electronic address aadhe, Cancer Genome Atlas Research N. integrated genomic characterization of pancreatic ductal adenocarcinoma. Cancer Cell. 2017;32:185-203 e13.

4. Bailey P, Chang DK, Nones K, Johns AL, Patch AM, Gingras $\mathrm{MC}$, et al. Genomic analyses identify molecular subtypes of pancreatic cancer. Nature. 2016;531:47-52.

5. Tuveson DA, Hingorani SR. Ductal pancreatic cancer in humans and mice. Cold Spring Harb Symp Quant Biol. 2005;70:65-72.

6. DuFort CC, DelGiorno KE, Hingorani SR. Mounting pressure in the microenvironment: fluids, solids, and cells in pancreatic ductal adenocarcinoma. Gastroenterology. 2016;150:1545-57 e2.
7. Singh M, Murriel CL, Johnson L. Genetically engineered mouse models: closing the gap between preclinical data and trial outcomes. Cancer Res. 2012;72:2695-700.

8. Morrison AH, Byrne KT, Vonderheide RH. Immunotherapy and prevention of pancreatic cancer. Trends Cancer. 2018;4:418-28.

9. Colvin EK, Scarlett CJ. A historical perspective of pancreatic cancer mouse models. Semin Cell Dev Biol. 2014;27:96-105.

10. Ponz-Sarvise M, Tuveson DA, Yu KH. Mouse models of pancreatic ductal adenocarcinoma. Hematol Oncol Clin North Am. 2015;29:609-17.

11. Behrens D, Walther W, Fichtner I. Pancreatic cancer models for translational research. Pharmacol Ther. 2017;173:146-58.

12. Shi C, Klein AP, Goggins M, Maitra A, Canto M, Ali S, et al. Increased prevalence of precursor lesions in familial pancreatic cancer patients. Clin Cancer Res. 2009;15:7737-43.

13. Brune K, Abe T, Canto M, O'Malley L, Klein AP, Maitra A, et al. Multifocal neoplastic precursor lesions associated with lobular atrophy of the pancreas in patients having a strong family history of pancreatic cancer. Am J Surg Pathol. 2006;30:1067-76.

14. Jiang F, Doudna JA. CRISPR-Cas9 structures and mechanisms. Annu Rev Biophys. 2017;46:505-29.

15. Wright AV, Nunez JK, Doudna JA. Biology and applications of CRISPR systems: harnessing nature's toolbox for genome engineering. Cell . 2016;164:29-44.

16. Zhang C, Quan R, Wang J. Development and application of CRISPR/Cas9 technologies in genomic editing. Hum Mol Genet. 2018;27(R2):R79-R88.

17. Hsu PD, Lander ES, Zhang F. Development and applications of CRISPR-Cas9 for genome engineering. Cell . 2014;157:1262-78.

18. Zhu Z, Li QV, Lee K, Rosen BP, Gonzalez F, Soh CL, et al. Genome editing of lineage determinants in human pluripotent stem cells reveals mechanisms of pancreatic development and diabetes. Cell Stem Cell. 2016;18:755-68.

19. Platt RJ, Chen S, Zhou Y, Yim MJ, Swiech L, Kempton HR, et al. CRISPR-Cas9 knockin mice for genome editing and cancer modeling. Cell . 2014;159:440-55.

20. Millette K, Georgia S. Gene editing and human pluripotent stem cells: tools for advancing diabetes disease modeling and beta-cell development. Curr Diab Rep. 2017;17:116.

21. Sanchez-Rivera FJ, Jacks T. Applications of the CRISPR-Cas9 system in cancer biology. Nat Rev Cancer. 2015;15:387-95.

22. Pawelczak KS, Gavande NS, VanderVere-Carozza PS, Turchi JJ. Modulating DNA repair pathways to improve precision genome engineering. ACS Chem Biol. 2018;13:389-96.

23. Bibikova M, Beumer K, Trautman JK, Carroll D. Enhancing gene targeting with designed zinc finger nucleases. Science. 2003;300:764.

24. Jasin M, de Villiers J, Weber F, Schaffner W. High frequency of homologous recombination in mammalian cells between endogenous and introduced SV40 genomes. Cell. 1985;43(3 Pt 2):695-703.

25. Rudin N, Sugarman E, Haber JE. Genetic and physical analysis of double-strand break repair and recombination in Saccharomyces cerevisiae. Genetics. 1989;122:519-34.

26. Chiou SH, Winters IP, Wang J, Naranjo S, Dudgeon C, Tamburini $\mathrm{FB}$, et al. Pancreatic cancer modeling using retrograde viral vector delivery and in vivo CRISPR/Cas9-mediated somatic genome editing. Genes \& Dev. 2015;29:1576-85.

27. Maresch R, Mueller S, Veltkamp C, Ollinger R, Friedrich M, Heid $\mathrm{I}$, et al. Multiplexed pancreatic genome engineering and cancer induction by transfection-based CRISPR/Cas9 delivery in mice. Nat Commun. 2016;7:10770.

28. Liu C, Zhang L, Liu H, Cheng K. Delivery strategies of the CRISPR-Cas9 gene-editing system for therapeutic applications. J Control Release: Off J Control Release Soc. 2017;266:17-26. 
29. Moreno AM, Fu X, Zhu J, Katrekar D, Shih YV, Marlett J, et al. In situ gene therapy via AAV-CRISPR-Cas9-mediated targeted gene regulation. Mol Ther. 2018;26:1818-27.

30. Kemaladewi DU, Maino E, Hyatt E, Hou H, Ding M, Place $\mathrm{KM}$, et al. Correction of a splicing defect in a mouse model of congenital muscular dystrophy type 1A using a homologydirected-repair-independent mechanism. Nat Med. 2017;23:984-9.

31. Tabebordbar M, Zhu K, Cheng JKW, Chew WL, Widrick JJ, Yan $\mathrm{WX}$, et al. In vivo gene editing in dystrophic mouse muscle and muscle stem cells. Science. 2016;351:407-11.

32. Chew WL, Tabebordbar M, Cheng JK, Mali P, Wu EY, Ng AH, et al. A multifunctional AAV-CRISPR-Cas9 and its host response. Nat Methods. 2016;13:868-74.

33. Kawaguchi Y, Cooper B, Gannon M, Ray M, MacDonald RJ, Wright CV. The role of the transcriptional regulator Ptfla in converting intestinal to pancreatic progenitors. Nat Genet. 2002;32:128-34.

34. Bardeesy N, Aguirre AJ, Chu GC, Cheng KH, Lopez LV, Hezel AF, et al. Both p16(Ink4a) and the p19(Arf)-p53 pathway constrain progression of pancreatic adenocarcinoma in the mouse. Proc Natl Acad Sci USA. 2006;103:5947-52.

35. Hruban RH, Klimstra DS. Adenocarcinoma of the pancreas. Semin Diagn Pathol. 2014;31:443-51.

36. Adsay NV, Basturk O, Cheng JD, Andea AA. Ductal neoplasia of the pancreas: nosologic, clinicopathologic, and biologic aspects. Semin Radiat Oncol. 2005;15:254-64.

37. Dawkins JB, Wang J, Maniati E, Heward JA, Koniali L, Kocher $\mathrm{HM}$, et al. Reduced expression of histone methyltransferases KMT2C and KMT2D correlates with improved outcome in pancreatic ductal adenocarcinoma. Cancer Res. 2016;76:4861-71.

38. Vonderheide RH. Tumor-promoting inflammatory networks in pancreatic neoplasia: another reason to loathe Kras. Cancer Cell. 2014;25:553-4

39. Bryant KL, Mancias JD, Kimmelman AC, Der CJ. KRAS: feeding pancreatic cancer proliferation. Trends Biochem Sci. 2014;39:91-100.

40. Livshits G, Alonso-Curbelo D, Morris JPt, Koche R, Saborowski M, Wilkinson JE, et al. Arid1a restrains Kras-dependent changes in acinar cell identity. eLife. 2018;7:e35216.

41. Kimura Y, Fukuda A, Ogawa S, Maruno T, Takada Y, Tsuda M, et al. ARID1A maintains differentiation of pancreatic ductal cells and inhibits development of pancreatic ductal adenocarcinoma in mice. Gastroenterology. 2018;155:194-209 e2.

42. Zhang ZM, Xiao S, Sun GY, Liu YP, Zhang FH, Yang HF, et al. The clinicopathologic significance of the loss of BAF250a (ARID1A) expression in endometrial carcinoma. Int J Gynecol Cancer. 2014;24:534-40.

43. Abe H, Hayashi A, Kunita A, Sakamoto Y, Hasegawa K, Shibahara $\mathrm{J}$, et al. Altered expression of AT-rich interactive domain
1A in hepatocellular carcinoma. Int $\mathrm{J}$ Clin Exp Pathol. 2015;8:2763-70.

44. Zhang L, Wang C, Yu S, Jia C, Yan J, Lu Z, et al. Loss of ARID1A expression correlates with tumor differentiation and tumor progression stage in pancreatic ductal adenocarcinoma. Technol Cancer Res Treat. 2018;17:1533034618754475.

45. Sahin F, Maitra A, Argani P, Sato N, Maehara N, Montgomery E, et al. Loss of Stk11/Lkb1 expression in pancreatic and biliary neoplasms. Mod Pathol. 2003;16:686-91.

46. Sato N, Rosty C, Jansen M, Fukushima N, Ueki T, Yeo CJ, et al. STK11/LKB1 Peutz-Jeghers gene inactivation in intraductal papillary-mucinous neoplasms of the pancreas. Am J Pathol. 2001;159:2017-22.

47. Hezel AF, Gurumurthy S, Granot Z, Swisa A, Chu GC, Bailey G, et al. Pancreatic LKB1 deletion leads to acinar polarity defects and cystic neoplasms. Mol Cell Biol. 2008;28:2414-25.

48. Lo B, Strasser G, Sagolla M, Austin CD, Junttila M, Mellman I. Lkb1 regulates organogenesis and early oncogenesis along AMPK-dependent and -independent pathways. J Cell Biol. 2012;199:1117-30.

49. Habbe N, Shi G, Meguid RA, Fendrich V, Esni F, Chen H, et al. Spontaneous induction of murine pancreatic intraepithelial neoplasia (mPanIN) by acinar cell targeting of oncogenic Kras in adult mice. Proc Natl Acad Sci USA. 2008;105:18913-8.

50. Gidekel Friedlander SY, Chu GC, Snyder EL, Girnius N, Dibelius G, Crowley D, et al. Context-dependent transformation of adult pancreatic cells by oncogenic K-Ras. Cancer Cell. 2009; 16:379-89.

51. Lee AYL, Dubois CL, Sarai K, Zarei S, Schaeffer DF, Sander M, et al. Cell of origin affects tumour development and phenotype in pancreatic ductal adenocarcinoma. Gut. 2018. Accessed on 23 January 2018. https://doi.org/10.1136/gutjnl-2017-314426.

52. Guerra C, Schuhmacher AJ, Canamero M, Grippo PJ, Verdaguer L, Perez-Gallego L, et al. Chronic pancreatitis is essential for induction of pancreatic ductal adenocarcinoma by K-Ras oncogenes in adult mice. Cancer Cell. 2007;11:291-302.

53. Chen M, Maeng K, Nawab A, Francois RA, Bray JK, Reinhard $\mathrm{MK}$, et al. Efficient gene delivery and expression in pancreas and pancreatic tumors by capsid-optimized AAV8 vectors. Hum Gene Ther Methods. 2017;28:49-59.

54. Smith LJ, Wright J, Clark G, Ul-Hasan T, Jin X, Fong A, et al. Stem cell-derived clade F AAVs mediate high-efficiency homologous recombination-based genome editing. Proc Natl Acad Sci USA. 2018;115:E7379-E88.

55. Mueller S, Engleitner T, Maresch R, Zukowska M, Lange $\mathrm{S}$, Kaltenbacher $\mathrm{T}$, et al. Evolutionary routes and KRAS dosage define pancreatic cancer phenotypes. Nature. 2018;554:62-8. 